\title{
The Impact of Subungual Osteochondroma Deformans on Severely Mycotic Toenails
}

\author{
Christopher Brian Stewart, Maryellen Ann Waltz \\ Central Virginia Foot \& Ankle Laser Center, Charlottesville, VA, USA \\ Email: drchrisstewart@cvillefootankle.com
}

How to cite this paper: Stewart, C.B. and Waltz, M.A. (2019) The Impact of Subungual Osteochondroma Deformans on Severely Mycotic Toenails. Journal of Cosmetics, Dermatological Sciences and Applications, 9, 56-65.

https://doi.org/10.4236/jcdsa.2019.91005

Received: January 31, 2019

Accepted: March 26, 2019

Published: March 29, 2019

Copyright $\odot 2019$ by author(s) and Scientific Research Publishing Inc. This work is licensed under the Creative Commons Attribution International License (CC BY 4.0).

http://creativecommons.org/licenses/by/4.0/

\begin{abstract}
Background: We examined 94 toenails exhibiting clinical signs of severe onychomycosis taking into account multiple contributing factors, not the least of which was Subungual Osteochondroma Deformans (SOD). We hypothesize that SOD plays a significant role in both the etiology and treatment of onychomycosis. Methods/Results: A retrospective post-treatment outcome analysis was performed comparing two groups of severely mycotic toenails. The first group of 25 fungal nails showed $54.31 \%$ improvement following treatment by both surgical nail avulsion and a 1064 YAG laser-based Nail Restoration algorithm. The second group of 69 fungal nails was not only treated using the same nail avulsion/laser-based algorithm but was also evaluated for SOD lesions which were surgically removed when present, resulting in $95.31 \%$ improvement. The overall incidence of SOD lesions in the second group of severely mycotic toenails was 73.91\%. Conclusion: We submit that at 73.91\% Subungual Osteochondroma Deformans has an under-reported and under-appreciated association with toenail fungus. Generally, we find that our success rates in treating severe toenail fungus using our laser-based Nail Restoration Protocols are much higher when SOD lesions are surgically removed.
\end{abstract}

\section{Keywords}

Subungual Osteochondroma Deformans, Onychomycosis, Laser, Toenail, Topical, Surgery, Bone Spur

\section{Background}

Fungal infection of the nail, onychomycosis, is the most common nail disease and is a frequent medical problem in adults. It has been reported that $20 \%$ of people between 40 and 60 years of age have this medical condition, while up to $90 \%$ of Americans over the age of 65 may have this infection [1]. A study on 
quality of life found that patients with confirmed onychomycosis experienced problems with nail-trimming, embarrassment, pain, nail pressure, and discomfort wearing shoes [2]. Additional findings were that onychomycosis interfered with social relationships. Subjects with toenail involvement sought medical care more frequently than those with fingernail involvement.

Onychomycosis is difficult to treat and historically $20 \%$ of patients with affliction of the toenail do not respond to therapy [3]. Remission after treatment is also rare and recurrence is common due to the difficulty in eradicating the pathogen [1]. Cure rates are low and treatment can extend over a lengthy period of time and can be expensive. The presence of dermatophytes, primarily Trichophyton rubrum, causes most of the superficial fungal infections [4]. Kemna et al. found that dermatophytes had caused $94.7 \%$ of tinea pedis cases and $81.9 \%$ of onychomycosis cases [4].

A study by Malay, Yi, Borowsky et al. (2009) discussed the variety of variables that significantly decreased the likelihood of cure rates such as the presence of yeast, hyperhidrosis, tobacco use, proximal involvement (lunula), and greater than 50\% involvement in the transverse width of the nail [5].

Traditionally, mycological culture has been commonly used to diagnose and determine therapeutic intervention [6]. Negative mycological results on culture do not necessarily rule out the presence of onychomycosis as cultures can be negative in $30 \%$ of cases [3]. False negative results may be a function of bacterial overgrowth masking the underlying infection or previous antimycotic use by the patient [4]. In a study of non-dermatophyte onychomycosis, false-negative results of fungal culture were found to occur in $25 \%$ of subjects [7]. Several studies found that nail plate biopsy using the periodic acid-Schiff stain (PAS) produced greater accuracy in the diagnosis of onychomycosis [8] [9]. One study suggested that a combination of culture and histopathological evaluation of the nail plate yielded greater accuracy of diagnosis since histopathology alone did not provide information about vitality of the fungi or accurate identification of the pathogen [10]. Accurate pathogen identification via PCR assay has demonstrated to be twice as sensitive as culture in identifying dermatophytes, thereby allowing organism identification to complement the high sensitivity of PAS (Periodic Acid-Schiff Stain)/Gomori Methenamine Silver/Fontana Masson Stain [11]. Regardless of the culture method, it must be underscored that all diagnostic results are limited by the accuracy of the specimen obtained.

Current treatments include topical applications, systemic medications, surgical procedures, and laser-based approaches. Topical antifungals are reported to be relatively ineffective for extensive disease as the drug does not often penetrate the nail plate [1]. Systemic antifungals were reported to require at least three months of treatment and can create drug and other adverse reactions [1]. Adverse reactions include hepatitis, headache, gastrointestinal upset, and rash [12]. Partial or total nail avulsion has also been used to treat onychomycosis permitting the removal of the affected portion of the nail [12]. Most physicians do not 
practice this technique because poor cosmetic results and reoccurrence of infection are common [4] [12]. In a study of 40 patients in 2007, Grover and colleagues reported that none of the severe cases of onychomycosis were cured by avulsion [13]. When treatments fail, unappealing nail dystrophy and pain can lead some sufferers to opt to undergo permanent removal of the offending nail via a matrixectomy procedure. Laser treatment tends not to be covered by health insurance since it is viewed as a cosmetic disease. In one study 249 subjects (97\%) stated that they would be willing to pay for a nail fungus treatment with an $80 \%$ cure rate, even if their insurance would not cover the cost [2].

Researchers have been using lasers to treat onychomycosis since the 1980s [14]. The original application of lasers in the treatment of nail fungus utilized the creation of holes in the nail plate to provide optimal access of topical solutions to the fungus [14]. Currently, most laser treatment protocols direct the laser energy through the nail plate itself without any visual disturbance to the keratin layers. Using this "non-invasive" approach Ortiz stated in 2014 that "early data are promising" [15]. Though several lasers are FDA approved for the treatment of onychomycosis it is important to note that this approval is only for the "temporary clearing" of mildly to moderately infected nails and not for a "cure" [16].

We have treated over 20,000 nails of all severities in our own clinic. It has been our experience that although laser treatment alone through mildly to moderately infected nail plates often improves the condition, it rarely, if ever, resolves cases of severe onychomycosis. We will present our data on mild to moderate cases of onychomycosis in a follow up article. For the purposes of discussing the potential reasons for our relative success in treating the most severe cases of toenail fungus we've chosen to limit this study to the application of our $\mathrm{Nd}$ : YAG laser-based surgical protocol on only severely infected and deformed toenails.

\section{SOD Lesion}

Although several advancements in the treatment of onychomycosis have been made over the years, it is still a truism that a highly efficacious, predictable treatment continues to challenge the medical community at large. In this article we discuss the Subungual Osteochondroma Deformans (SOD) lesion as it relates to onychomycosis and we propose that by addressing this lesion, treatment outcomes can be greatly improved.

We define the Subungual Osteochondroma Deformans (SOD) lesion as a firm subungual growth diagnosed in the presence of onychomycosis. This direct relationship with onychomycosis distinguishes SOD lesions from a simple exostosis which can be diagnosed on plain radiographs and may or may not be symptomatic [17]. There must be nail plate fungal infection for an exostosis to be considered a SOD lesion. SOD lesions are comprised of bone, cartilage or a combination of bone and cartilage extending from the dorsal aspect of the distal phalanx of the digit. This condition is thought to arise from genetics, trau- 
ma or digital recurvatum of the distal phalanx. When large enough, SOD lesions can cause the nail plate to separate from the nailbed leading to mild, moderate or severe white streaking, curvature of the nail plate and opportunistic fungal infection. In advanced cases, the presence of SOD is often associated with moderate to severe dystrophy (Deformans), painful ingrown toenails, loosening of the nail plate, and even auto-avulsion of some or all of the nail plate. We have observed, during the past 15 years in treating toenail fungus, that SOD lesions, when not removed, may at least partially explain the failure of simple nail avulsions [13] to yield healthy new nail growth. SOD lesions do not typically allow for reattachment of the new nail plate to the nailbed tissue. The inability of a nail to reattach or "seal" can lead to poor cosmetic results and lysis. Lysis, defined as separation of the nail from the nailbed tissue, can act as a "dead space" which allows for fungus or other pathogens to enter the nail-nailbed interface.

We have isolated three SOD variants that occur in the presence of onychomycosis: Type 1-Bone only (traditional subungual exostosis); Type 2-Cartilage only (chondroma) and Type 3-Bone and Cartilage in combination (Osteochondro$\mathrm{ma})$. It has been our experience that when present, all three variants have the potential to adversely impact onychomycosis treatment outcomes.

\section{Patients and Methods}

This study retrospectively compares outcomes between two groups of patients with severe pedal onychomycosis. The treatment group of 25 severely mycotic nails consisted of 14 male and 10 females, while the treatment group of 69 severely mycotic nails consisted of 10 males and 26 females. Inclusion criteria for both groups of patients include: adult individuals with chief complaint of visual nail texture and color changes typical of severe onychomycosis, patients compliant with our protocol, healthy individuals with palpable pedal pulses, and either no history of diabetes, or well-controlled diabetes. Following third party ethical clearance, the first group of 25 severely mycotic toenails was treated using our Nd: YAG laser-based algorithm combined with avulsion of each toenail and patient applied daily antifungal/anti-inflammatory lubricant $\left(\right.$ NovoNail ${ }^{\circledR}$ Solution). The second group of 69 toenails were not only treated with the same laser-based surgical and topical NovoNail ${ }^{\mathbb{B}}$ Solution algorithm, but were also assessed for the presence of SOD lesions which were removed when present. The SOD lesions were excised via both blunt and sharp dissection down to bone using a combination of cross-cutting bone rasp and curettage instrumentation depending on size, composition (bone, cartilage, or combination thereof), location of the lesion. Both treatment groups applied the NovoNail Solution ${ }^{\circledR}$ for the entire duration of nail regrowth, which although varied from patient to patient, averaged 12 months.

The final results comparing each pre-treated nail to each post-treated nail were summarized using digital planimetry (Figure 3(a) and Figure 3(b)). Pla- 
nimetry is the process by which percent reduction in nail discoloration is expressed using a two-dimensional area over time.

\section{Results}

\section{Analysis of 25 Fungal Nails Not Accounting for SOD Lesions}

A retrospective analysis was performed on 25 severely mycotic toenails treated with surgical nail avulsion and our $1064 \mathrm{Nd:YAG} \mathrm{laser-based} \mathrm{surgical} \mathrm{algorithm}$ and daily application of topical NovoNail ${ }^{\circledR}$ Solution over a 12 -month period not taking into account the presence or absence of an SOD lesion in each toe.

Improvement across all 25 toenails was $54.81 \%$ as measured by digital planimetry. Analysis of 69 Fungal Nails Accounting for SOD Lesions

A retrospective analysis was performed on 69 severely mycotic toenails treated with surgical nail avulsion and our $1064 \mathrm{Nd}$ :YAG laser-based surgical algorithm and daily application of topical NovoNail ${ }^{\circledR}$ Solution over a 12 -month period evaluating for the presence or absence of an SOD lesion in each toe. When present, all SOD lesions were surgically removed. The incidence of Subungual Osteochondroma Deformans was 51/69 toes (73.91\%). This was determined via radiography, physical palpation of the nail bed and intraoperative visual confirmation. These nails were also rated using digital picture planimetry both before and after treatment (Figures 1-6).

The 69 Toenails treated in the case series where SOD lesions were identified and removed where present showed a $95.31 \%$ improvement in nail appearance following treatment.

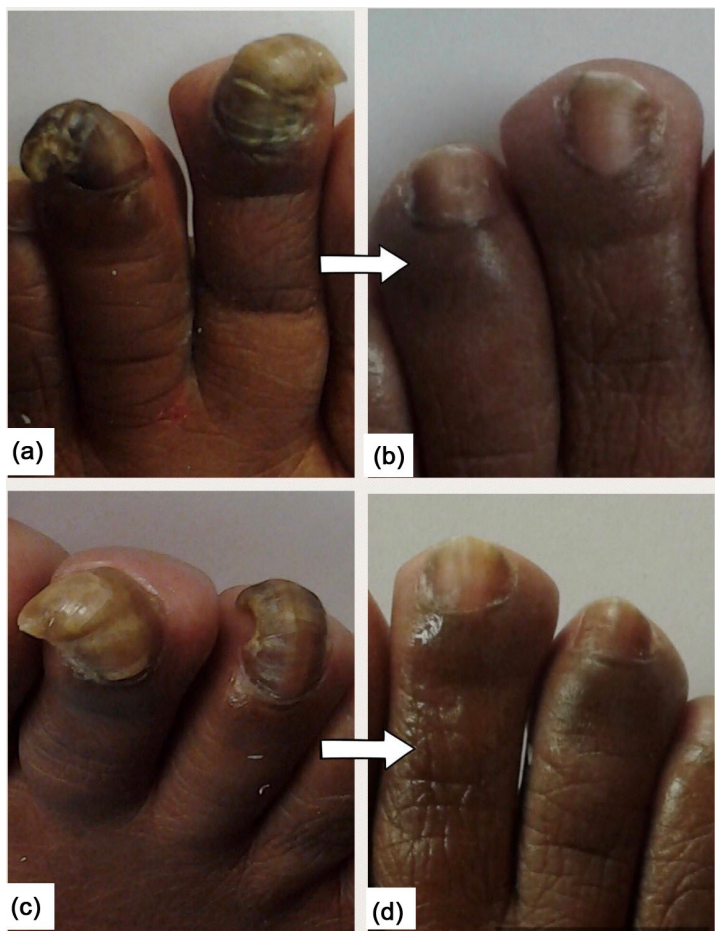

Figure 1. (a) and (c) Depiction of before nail restoration surgical procedure with excision of SOD; (b) and (d) Final photograph 12 months after completion of nail restoration. 


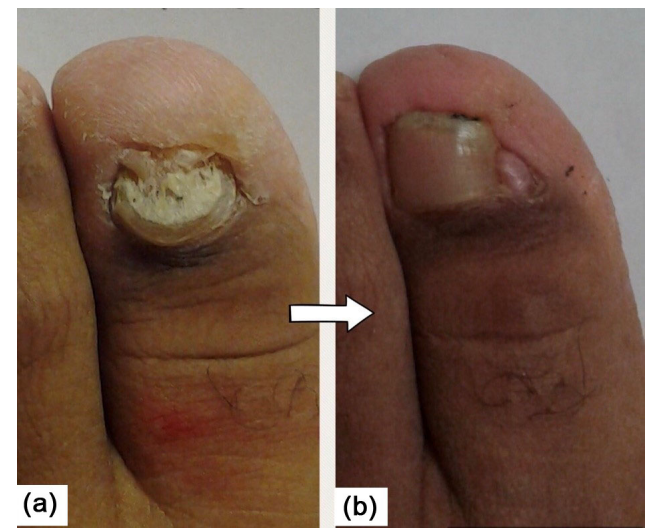

Figure 2. (a) and (b) Clinical photograph of severely dystrophic nail with evidence of SOD pre- and post-operatively following nail restoration surgical procedure.
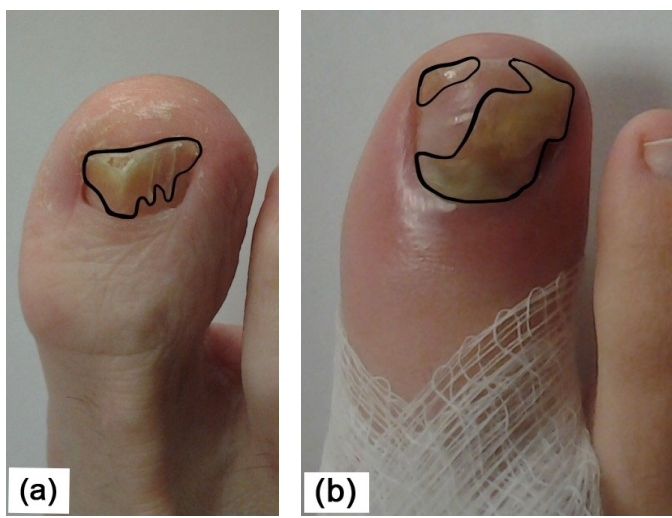

Figure 3. (a) and (b) Depiction of surface area affected by onychomycosis as analyzed via planimetry prior to nail restoration.
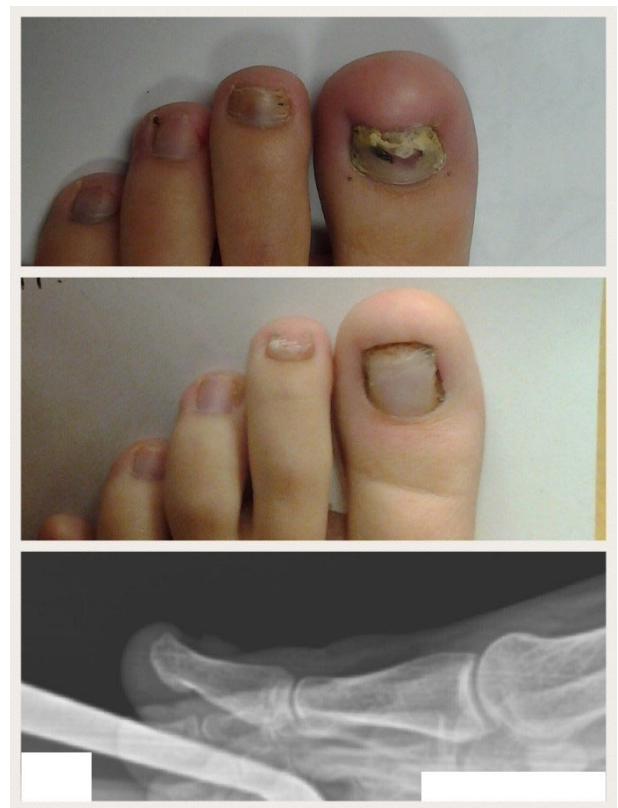

Figure 4. Pre-operative severe onychomycosis with SOD type III lesion (L1); pictured below: 12-month post-operative nail restoration with SOD excision. Last photograph depicting pre-operative lateral radiograph with SOD lesion. 


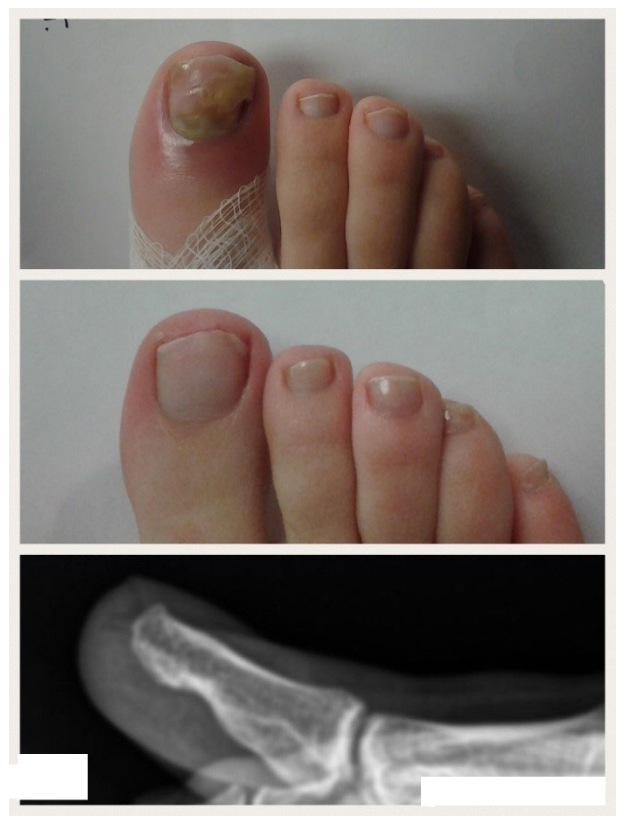

Figure 5. Pre-operative severe onychomycosis with SOD type I lesion (R1); pictured below: 12-month post-operative nail restoration with SOD excision. Last photograph depicting pre-operative lateral radiograph with SOD lesion.

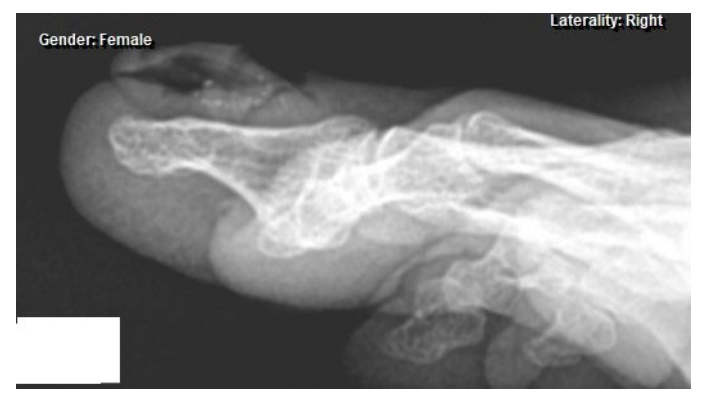

Figure 6. SOD type II lesion demonstrating cartilaginous lesion only with severe deformation of the nail plate visible on lateral toe radiograph.

\section{Discussion}

We compared the treatment of two groups of severely mycotic toenails where the main difference was the removal of Subungual Osteochondroma Deformans lesions when present. These findings support our experience that removal of SOD lesions in severely mycotic toenails greatly improves overall treatment outcomes.

In 2007 Grover reported a 0\% improvement using simple nail avulsion for severely mycotic toenails (16). We calculated a $54.81 \%$ improvement using our surgical laser-based algorithm in addition to nail avulsion and compliant patient daily application of NovoNail ${ }^{\circledR}$ Solution. Furthermore, and most significantly, we calculated a $95.31 \%$ improvement when this same protocol was combined with removal of all SOD lesions when present (Figure 7).

Our experience to date finds that the majority of SOD lesions are of the Type 3 variant which presents as an osseous spur with a cartilaginous cap. Plain 


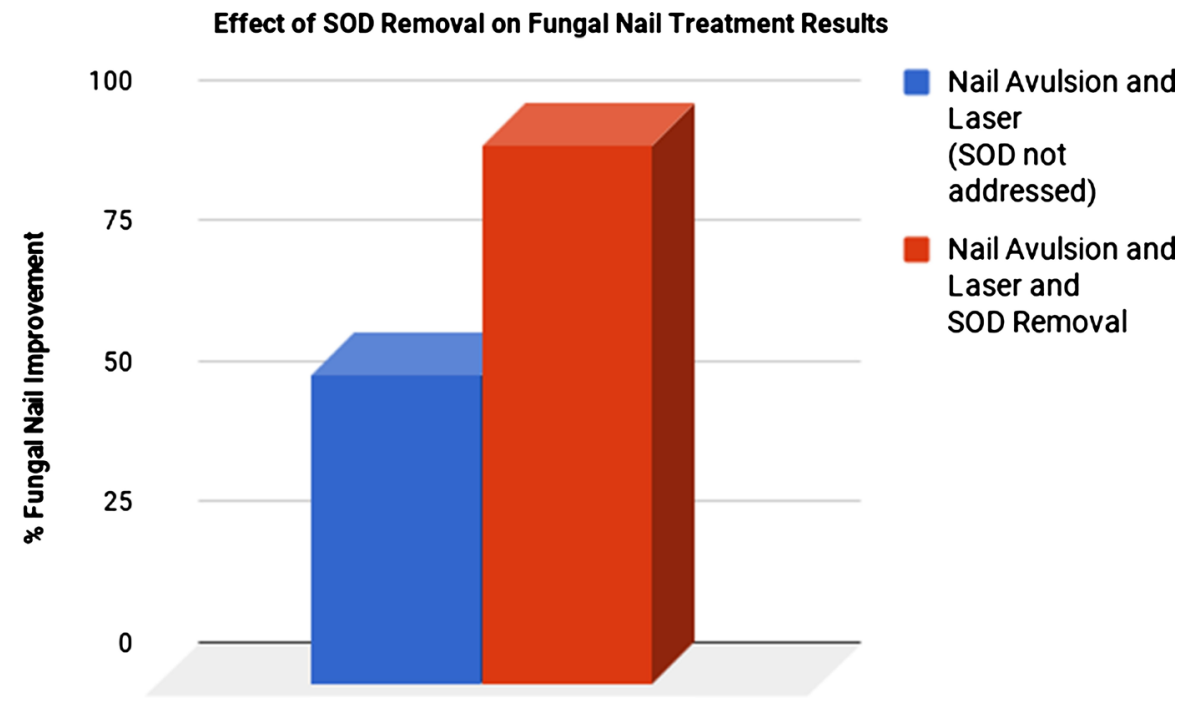

Figure 7. Comparison of fungal nail improvement with and without removal of the SOD lesion.

radiographs provide some assistance in diagnosing these lesions but often underrepresent the size of the spur due to the contribution of the cartilaginous component (Figure 4).

Though not as prevalent as Type 3, the radiographically "invisible" Type 2 purely cartilaginous SOD variant is not to be underestimated (Figure 6). These growths do not show up on plain radiographs yet can be just as deforming a force to the nail plate as the traditional subungual exostosis.

All in all, 51 out of the 69 nails reviewed in our second group had confirmed SOD lesions (73.91\%). This percentage, nearly 3 out of 4 , is much higher than the "uncommon" incidence of digital subungual exostosis reported in the general population [17]. We attribute the increased incidence of Subungual Osteochondroma Deformans to the correlative relationship these lesions appear to have with severe onychomycosis.

Fungal nail research is challenging for many reasons. The slow rate of nail regrowth makes developing successful treatment protocols a long and arduous process. In addition, the only true test for mycologic cure is to avulse and analyze the entire nail plate post treatment. Since this is a cosmetic procedure, we have found patients reluctant to undergo a complete avulsion after waiting 6 - 12 months for their new nail to grow in. For this reason and due to the relatively unreliable analyses of culture specimens, we, and many others, rely largely upon visual analysis using measures such as thickness and planimetry (percent reduction in nail discoloration over time). Future research building upon the relationship between SOD lesions and onychomycosis could include prospective studies that may or may not identify patients who are willing to donate their entire nail plate for mycologic analysis post treatment. We also recognize the potential for bias in this private practice generated retrospective study, despite efforts to minimize the potential for bias. Future studies may limit or remove such potential bias through double blind procedures and methods. 


\section{Conclusion}

The findings of Subungual Osteochondroma Deformans (SOD) lesions beneath the nail pate correlate highly with advanced pedal onychomycosis. 51 severely mycotic toenails out of a total of 69 demonstrated the presence of SOD (73.91\%). Furthermore, when removal of SOD was combined with a multifaceted laser-based treatment algorithm greater than $95.31 \%$ improvement was achieved.

\section{Conflicts of Interest}

The authors declare Dr Christopher B. Stewart is the founder of Novonail, LLC.

\section{References}

[1] Jayatilake, J.A.M.S., Tilakaratne, W.M. and Panagoda, G.J. (2009) Candidal Onychomycosis: A Mini-Review. Mycopathologia, 168, 165-173.

https://doi.org/10.1007/s11046-009-9212-X

[2] Drake, L.A., Scher, R.K., Smith, E.B., Faich, G.A., Smith, S.L., Hong, J.J. and Stiller, M.J. (1998) Effect of Onychomycosis on Quality of Life. Journal of the American Academy of Dermatology, 38, 702-704. https://doi.org/10.1016/S0190-9622(98)70199-9

[3] Iorizzo, M., Piraccini, B.M. and Tosti, A. (2007) New Fungal Nail Infections. Current Opinion in Infectious Diseases, 20, 142-145. https://doi.org/10.1097/QCO.0b013e32802ef47e

[4] Kemna, M.E. and Elewski, B.E. (1996) A U.S. Epidemiologic Survey of Superficial Fungal Diseases. Journal of the American Academy of Dermatology, 35, 539-542. https://doi.org/10.1016/S0190-9622(96)90675-1

[5] Malay, D.S., Yi, S., Borowsky, P., Downey, M.S. and Mlodzienski, A.J. (2009) Efficacy of Debridement Alone Versus Debridement Combined with Topical Antifungal Nail Lacquer for the Treatment of Pedal Onychomycosis: A Randomized, Controlled Trial. The Journal of Foot and Ankle Surgery, 48, 294-308. https://doi.org/10.1053/j.jfas.2008.12.012

[6] Elewski, B.E. (1995) Clinical Pearl: Diagnosis of Onychomycosis. Journal of the American Academy of Dermatology, 32, 500-501. https://doi.org/10.1016/0190-9622(95)90075-6

[7] Shemer, A., Davidovici, B., Grunwald, M.h., Trau, H. and Amichai, B. (2009) New Criteria for the Laboratory Diagnosis of Nondermatophyte Moulds in Onychomycosis. British Journal of Dermatology, 160, 37-39. https://doi.org/10.1111/j.1365-2133.2008.08805.x

[8] Weinberg, J.M., Koestenblatt, E.K. and Jennings, M.B. (2005) Utility of Histopathologic Analysis in the Evaluation of Onychomycosis. Journal of the American Podiatric Medical Association, 95, 258-263. https://doi.org/10.7547/0950258

[9] Karimzadegan-Nia, M., Mir-Amin-Mohammadi, A., Bouzari, N. and Firooz, A. (2007) Comparison of Direct Smear, Culture and Histology for the Diagnosis of Onychomycosis. Australasian Journal of Dermatology, 48, 18-21. https://doi.org/10.1111/j.1440-0960.2007.00320.x

[10] Weinberg, J.M., Koestenblatt, E.K., Tutrone, W.D., Tishler, H.R. and Najarian, L. (2003) Comparison of Diagnostic Methods in the Evaluation of Onychomycosis. Journal of the American Academy of Dermatology, 49, 193-197. https://doi.org/10.1067/S0190-9622(03)01480-4 
[11] (2017) Molecular Genetic Testing (PCR Assay) Now Available for Rapid Fungal Identification.

[12] Baran, R., Hay, R.J. and Garduno, J.I. (2008) Review of Antifungal Therapy and the Severity Index for Assessing Onychomycosis: Part I. Journal of Dermatological Treatment, 19, 72-81. https://doi.org/10.1080/09546630701243418

[13] Grover, C., Bansal, W., Nanda, S., et al. (2007) Combination of Surgical Avulsion and Topical Therapy for Single Nail Onychomycosis: A Randomized Controlled Trial. British Journal of Dermatology, 157, 364-368. https://doi.org/10.1111/j.1365-2133.2007.08014.x

[14] Bristow, I.R. (2014) The Effectiveness of Lasers in the Treatment of Onychomycosis: A Systematic Review. Journal of Foot and Ankle Research, 7, 34.

[15] Ortiz, A.E., Avram, M.M. and Wanner, M.A. (2014) A Review of Lasers and Light for the Treatment of Onychomycosis. Lasers in Surgery and Medicine, 46, 117-124. https://doi.org/10.1002/lsm.22211

[16] Vlahovic, T. (2016) Recent Research Shows the Failure of Laser Therapy as a Monotherapy for Onychomycosis. Podiatry Today, 29, 14.

[17] Lipner, S., Scher, R. and Ashourian, N. (2016) Subungual Exostosis (Dupuytren's Subungual Exostosis, Enchondroma). The Clinical Advisor. Decision Support in Medicine. 